\title{
Clinically suspect arthralgia - are we going towards a new shift in the therapeutic paradigm of rheumatoid arthritis?
}

\author{
Catalina Elena Ionescu ${ }^{1,2}$, Mihaela Agache ${ }^{1,2}$, Claudiu Popescu ${ }^{1,2}$, Luminita Enache ${ }^{1,2}$, \\ Corina Mogosan ${ }^{1,2}$, Catalin Codreanu ${ }^{1,2}$ \\ 1 "Dr. Ion Stoia" Clinical Center for Rheumatic Diseases, Bucharest, Romania \\ 2"Carol Davila" University of Medicine and Pharmacy, Bucharest, Romania
}

\begin{abstract}
Background. There is a time sensitive window of opportunity in rheumatoid arthritis (RA) in which therapeutic intervention is more effective, the disease being more susceptible to the immunomodulatory effects of the remissive medication. The goal is to prevent osteo-articular damage, which causes severe functional deficit, and to raise the chance to lead the disease in remission. Evolution towards RA represents a multi-step process. In other medical fields prevention has the same important role as treatment, so could we in the future switch again the therapeutic paradigm in RA, from early treatment to prevention of RA, by treating patients with high risk of developing disease? Initiating treatment in the pre-RA phases could potentially lead to a better immune modulation or even preventing disease development by acting on less mature pathogenic processes. Treating in the initial symptomatic phase of the disease could potentially be more effective in reducing disease persistence and the development of structural lesions. The clinically suspect arthralgia (CSA) definition offers a support of clinical parameters for future longitudinal studies, where together with para clinical parameters, laboratory studies and imagistic studies, could lead to the development of imminent RA classification criteria. Currently there are more ongoing studies that have the primary objective to prove this concept with different subpopulations and treatments, but most of them have inclusion criteria based on the presence of autoantibodies. The publication of this trials results in the next decade will help to better understand the efficacy of therapeutic intervention with the scope of preventing chronic arthritis and what subset of patients at risk to treat.

There are no recommendations for management of CSA, but current practice is symptomatic treatment with nonsteroidal anti-inflammatory drugs, pain relievers and of course monitoring.
\end{abstract}

Keywords: clinically suspect arthralgia, window of opportunity, pre-arthritis phase

\section{BACKGROUND}

Rheumatoid arthritis (RA) is an immune mediated systemic inflammatory disease, which predominantly affects the synovial joints, where it determines a chronic inflammatory process, which leads to early, progressive, irreversible osteo-articular damage, with significant functional deficit. Is frequently associated with significant extra-articular systemic manifestations, which threw complications, lead to reduced life expectancy with 5-10 years. RA is the most frequent form of inflammatory rheumatological disease with a population prev- alence of $0.5-1 \%$ with major socio-economic consequences [1].

It's destructive nature, the significant functional deficit and the severe damage over quality of life of the patients with rheumatoid arthritis are the main reasons why major efforts have been submitted towards better understanding disease pathogeny, early recognition and diagnostic, using the ACR-EULAR classification criteria, developed in 2010 [2], with the purpose of starting treatment as early as possible, developing new disease modifying antirheumatic drugs and elaborating treat to target and tight 
control recommendations for management, the last update was made in 2019.

It has been clearly demonstrated that there is a time sensitive window of opportunity in which therapeutic intervention is more effective, the disease being more susceptible to the immunomodulatory effects of the remissive medication. The goal is to prevent osteo-articular damage, which causes severe functional deficit, and to raise the chance to lead the disease in remission. Current disease modifying antirheumatic drugs are efficient in suppressing inflammation, but their ability to modify the persistent nature of the disease is limited [3].

A study developed in 2011 on early rheumatoid arthritis patients, disease under 12 months, treated with a tight control protocol, which objectives were to determine clinical remission predictors and predictors of no radiographic progression, has shown that very early rheumatoid arthritis, disease under 12 weeks, is an independent ACR remission predictor and after 12 months is the only factor which predicts lack of erosions [4].

In the last couple of years, studies have shifted their focus on the earliest phases in disease development. This is how we now know that rheumatoid arthritis is preceded by the development of autoantibodies, Ig M rheumatoid factors and anti-citrullinated protein antibodies, with a median period of 5-10 years, and also by the presence of acute phase reactants. The subjects with arthralgia and presence of autoantibodies have an approximative risk of $30 \%$ to develop RA [5].

Evolution towards RA represents a multi-step process. The European League Against Rheumatism (EULAR) study group has differentiated the next phases in rheumatoid arthritis development: genetic risk factors for RA; environmental risk factors for RA; systemic autoimmunity associated with RA; symptoms without clinical arthritis (clinically suspect arthralgia CSA); unclassified arthritis and finally RA. The pre-RA terminology will be exclusively used for patients who develop RA. In is important to understand that the pre-RA phases don't necessarily respect this chronology and not all patients will pass through all this stages [6].

In other medical fields prevention has the same important role as treatment, so could we in the future switch again the therapeutic paradigm in RA, from early treatment to prevention of RA, by treating patients with high risk of developing disease?

Initiating treatment in the pre-RA phases could potentially lead to a better immune modulation or even preventing disease development by acting on less mature pathogenic processes [6].

\section{CLINICALLY SUSPECT ARTHRALGIA}

Patients with clinically suspect arthralgia present articular symptoms without any signs of arthritis and they are considered at high risk to develop RA. Identifying high risk patients, clinically suspect arthralgia patients, used to be based on the clinical expertise of the rheumatologist.

The symptomatic phase, CSA, which precedes clinical arthritis could represent the first opportunity to identify patients at risk for imminent RA. It has been proven that early initiation of DMARDs in RA is more efficient in modulating the erosive and persistent nature of the disease [7]. Treating in the initial symptomatic phase of the disease could potentially be more effective in reducing disease persistence and the development of structural lesions.

To study this hypothesis there was the need for homogenous patient population. Recent data suggest that of all the patients presenting with arthralgia CSA represent only a small percentage of $7 \%$. And from all CSA cases only $20 \%$ will later develop RA [8]. The clinical expertise of rheumatologists has been sufficiently precise to differentiate arthralgia at high risk for imminent RA from other types of arthralgia. In clinical practice we could have used the CSA concept, but its subjectivity raised the need to define a phenotype.

To study the hypothesis in which treatment in the CSA phase is more effective there was the need for homogenous study patient population, so the EULAR study group defined the CSA phenotype in 2016 [9].

CSA has been defined by using seven domains, five from the patient's history taking and two from the clinical examination. We have to select patients with arthralgia, without any signs of clinical arthritis and without any other evident causes for joint pain. From the patient's history taking we have 5 domains: joint symptomatology of recent onset, duration under one year, symptoms located in the metacarpophalangeal joints, duration of morning stiffness over 60 minutes, most severe symptoms presenting in the early morning and the presence of a first degree relative with RA. From the clinical exam there are two domains: difficulty with making a fist and positive squeeze test of the metacarpophalangeal joints [10].

The CSA definition offers a support of clinical parameters for future longitudinal studies, where together with para clinical parameters, laboratory studies and imagistic studies, could lead to the development of imminent RA classification criteria. Future observational and interventional prospective studies could beneficiate of sensitive inclusion criteria thanks to the CSA definition, and the addition of laboratory and imagistic investigations, progression markers for RA, would offer specificity [9]. 


\section{TREATMENT IN THE PRE-ARTHRITIS PHASE}

Experimental studies on murine models of arthritis suggest that initiating treatment before arthritis development could be more effective. A meta-analysis from 2017 of 16 studies has proven that initiating DMARD therapy in the induction phase of arthritis, before the development of clinical arthritis and of autoimmunity, had beneficial effects on the severity of the arthritis. The most solid evidence was for methotrexate and abatacept [10]. Treating has been effective even on murine models with antibodies presence, but without clinical arthritis. There are no head-to-head trials, but at this subtype, murine models with antibody presence, methotrexate seemed more effective than anti-tumor necrosis factor blockers. These experimental studies have had a lot of limitations, the most relevant being the fact that the treatment period has been extended in the clinical arthritis phase and the primary objective was arthritis severity, not arthritis development. The trend in this animal studies favors initiating treatment in the pre-arthritis phase, but there is the need for bigger head-to-head studies, limited on the pre-arthritis phase period, in which different treatments, like methotrexate and abatacept be compared in order to obtain more valuable information [11].

In 2009, the first placebo-controlled trial, that evaluated initiating treatment in the pre-arthritis phase, was published and it demonstrated that two intramuscular injections of dexamethasone in seropositive arthralgia patients, decreased autoantibody levels but did not prevent the development of arthritis [12].

In 2016, the PRAIRI trial (prevention of clinically manifest RA by B cell directed therapy in the earliest phase of the disease) demonstrated that a single infusion of rituximab in seropositive patients with arthralgia and any sign of systemic and/or local inflammation delayed, but did not prevent, the development of clinical arthritis [13].

Currently there are more ongoing studies that have the primary objective to prove this concept with different subpopulations and treatments, but most of them have inclusion criteria based on the presence of autoantibodies. The publication of this trials results in the next decade will help to better understand the efficacy of therapeutic intervention with the scope of preventing chronic arthritis and what subset of patients at risk to treat.

There are no recommendations for management of CSA, but current practice is symptomatic treatment with non-steroidal anti-inflammatory drugs, pain relievers and of course monitoring [11].

The APPIPRA study (arthritis prevention in the pre-clinical phase of RA with abatacept: a multi-center, randomized, double-blind, parallel-group, placebo-controlled clinical trial protocol) included patients with non-traumatic arthralgia who are auto-antibody positive (either positive for RF and ACPA or have high levels of ACPA) had as a primary outcome the development of either clinical arthritis or RA. The intervention was represented by Abatacept $125 \mathrm{mg}$ weekly over twelve months [14].

The ARIAA trial, had the same intervention, Abatacept weekly over six months, and included patients who are positive for ACPA and have subclinical inflammation in the dominant hand, detected by MRI. The primary end point was improvement of inflammation [11].

TREAT Early Arthralgia to Reverse or Limit Impending Exacerbation to Rheumatoid arthritis (TREAT EARLIER): a randomized, double-blind, placebo-controlled clinical trial protocol included patients with CSA and recent-onset arthralgia $(<1$ year) that is suspect to progress to RA according to the expertise of the treating rheumatologist and need to have subclinical inflammation of the hand or foot joints at $1.5 \mathrm{~T}$ MRI. Intervention will be randomly assigned and includes a single-dose of intramuscular $120 \mathrm{mg}$ methylprednisolone followed by methotrexate (increasing dose to $25 \mathrm{mg} /$ week orally) or placebo (both; injection and tablets) over the course of 1 year. Thereafter, participants are followed for another year. The primary endpoint is the development of clinically detectable arthritis, either fulfilling the 2010 criteria for RA or unclassified clinical arthritis of $\geq 2$ joints, which persists for at least 2 weeks. DMARD-free status is a co-primary endpoint. It will test the hypothesis whether intervention in patients in this early phase with the cornerstone treatment of classified RA (methotrexate) hampers the development of persistent RA and reduce the disease burden of RA [15].

The STAtins to Prevent Rheumatoid Arthritis (STAPRA) trial included auto-antibody positive patients who received atorvastatin $40 \mathrm{mg}$ daily for 36 months had as a primary outcome the development of clinically detectable arthritis. the trial was prematurely stopped due to a low inclusion rate, mainly because of an unwillingness to participate [16].

In 2016, the StopRA trial begun. It included ACPA positive patients without inflammatory arthritis, who were recruited from health fairs or rheumatology clinics and who were first degree relatives of patients with RA. The intervention was hydroxychloroquine 200-400 mg a daily, over 12 months. The outcome was represented by development of clinically apparent RA.

Risk stratification is essential in order to advance studies in preventing RA. Adequate risk stratification is crucial in the design and interpretation of prevention studies. The trials strength is considerably influenced by each individual's risk from the 
population study to reach the primary objective, clinical evident RA. The higher the percentage of patients at low risk of developing RA, the lower the strength of the study will be, especially in the case of a small group.

An illustrative example of the importance of risk stratification was provided by the post-hoc analysis of the PROMPT study (probable AR: methotrexate versus placebo).

Patients with undifferentiated arthritis (UA) received MTX or placebo, the primary endpoint was RA prevention and the secondary endpoint was remission without medication. Analysis of the entire group showed that MTX neither prevented RA, nor led to remission without medication. The initial post-hoc analysis stratified patients after the presence of ACPA and showed the efficacy of MTX in seropositive versus seronegative patients. Seropositive patients had a higher risk of developing RA, but stratification based on ACPA alone was too simplistic, which is why investigators developed and validated a model to predict the risk of an individual with UA to develop RA based on clinical features, the presence of rheumatoid factors, ACPA and C-reactive protein (PCR). When repeating the analysis considering only patients at high risk of developing RA, over $80 \%$ in the following year, MTX appeared to prevent the development of RA with a NNT of 2 [17,18].

In addition to ACPA with a positive predictive value of up to $63 \%$ for patients with CSA to develop RA, it should be noted that $50 \%$ of RA patients are ACPA negative, and other potential biomarkers in predicting progression to RA would be by detecting subclinical joint inflammation, by ultrasound or magnetic resonance imaging. Studies are needed to directly compare the predictive accuracy of both imaging modalities and to evaluate the minimum region to be explored with maximum results, taking

\section{REFERENCES}

1. Codreanu C. Artrita reumatoidă și boli înrudite. In: Ionescu R. Reumatologie - Curs Universitar. București: Editura Universitară "Carol Davila”, 2017:136-171.

2. Kay J, Upchurch KS. ACR/EULAR 2010 rheumatoid arthritis classification criteria. Rheumatology (Oxford). 2012;51(Suppl 6):vi5-9.

3. Gwinnutt JM, Symmons DPM, MacGregor AJ, Chipping JR, Marshall T, Lunt M, et al. Twenty-Year Outcome and Association Between Early Treatment and Mortality and Disability in an Inception Cohort of Patients With Rheumatoid Arthritis: Results From the Norfolk Arthritis Register. Arthritis Rheumatol. 2017;69(8):1566-1575.

4. Bosello S, Fedele AL, Peluso G, Gremese E, Tolusso B, Ferraccioli G. Very early rheumatoid arthritis is the major predictor of major outcomes: clinical ACR remission and radiographic nonprogression. Ann Rheum Dis. 2011;70(7):1292-1295.

5. van de Stadt $L A$, van der Horst $A R$, de Koning $M H$, Bos $W H$, Wolbink GJ, van de Stadt RJ, et al. The extent of the anti- into account that ultrasound is an affordable but time-consuming method, and MRI has high costs. Subclinical inflammation predicts development to RA, independent of antibody status and clinical characteristics in patients with CSA, indicating that the use of both antibodies and imaging methods could further increase the risk of developing RA compared to the independent use of each biomarker. In these patients, elevated CRP may also independently predict the risk of developing RA [11].

In RA, the balance of treatment or not is not new, 15 years ago similar discussions were related to patients with UA, clinically evident arthritis, who did not meet the classification criteria for RA. Only after extensive validation of risk prediction models with acceptable accuracy there were recommendations of management for treatment of UA made. Because UA treatment is now a common practice, it is not known what the balance is in terms of treatment, nor the natural evolution of UA [18].

\section{CONCLUSIONS}

Although clinically suspected arthralgia may be part of the therapeutic opportunity window of RA, descriptive observational studies are needed to assess the natural evolution of this stage and help us to develop criteria classification for imminent RA, so that we can know with the highest certainty which patients to treat, in order to avoid both overdiagnosis and excessive treatment, but also to be able to potentially prevent the progression of the disease in high-risk patients.

Most of the studies had inclusion criteria for clinically suspect arthralgia, according to the rheumatologist opinion, while there is the need for study designs with inclusion criteria represented by clinically suspect arthralgia, according to the EULAR definition. citrullinated protein antibody repertoire is associated with arthritis development in patients with seropositive arthralgia. Ann Rheum Dis. 2011;70(1):128-133.

6. Gerlag DM, Raza K, van Baarsen LG, Brouwer E, Buckley CD, Burmester GR, et al. EULAR recommendations for terminology and research in individuals at risk of rheumatoid arthritis: report from the Study Group for Risk Factors for Rheumatoid Arthritis. Ann Rheum Dis. 2012;71(5):638-641.

7. Finckh $\mathrm{A}$, Liang $\mathrm{MH}$, van Herckenrode $\mathrm{CM}$, de Pablo P. Longterm impact of early treatment on radiographic progression in rheumatoid arthritis: A meta-analysis. Arthritis Rheum. 2006;55(6):864-872.

8. van Steenbergen HW, Mangnus L, Reijnierse M, Huizinga TW, van der Helm-van Mil AH. Clinical factors, anticitrullinated peptide antibodies and MRI-detected subclinical inflammation in relation to progression from clinically suspect arthralgia to arthritis. Ann Rheum Dis. 2016;75(10):1824-1830. 
9. van Steenbergen HW, Aletaha $D$, Beaart-van de Voorde LJ, Brouwer E, Codreanu C, Combe B, et al. EULAR definition of arthralgia suspicious for progression to rheumatoid arthritis. Ann Rheum Dis. 2017;76(3):491-496.

10. Dekkers JS, Schoones JW, Huizinga TW, Toes RE, van der Helmvan Mil AH. Possibilities for preventive treatment in rheumatoid arthritis? Lessons from experimental animal models of arthritis: a systematic literature review and meta-analysis. Ann Rheum Dis. 2017;76 (2):458-467.

11. van Steenbergen HW, da Silva JAP, Huizinga TWJ, van der Helm-van Mil AHM. Preventing progression from arthralgia to arthritis: targeting the right patients. Nat Rev Rheumatol. 2018;14(1):32-41.

12. Bos WH, Dijkmans BA, Boers $M$, van de Stadt RJ, van Schaardenburg D. Effect of dexamethasone on autoantibody levels and arthritis development in patients with arthralgia: a randomised trial. Ann Rheum Dis. 2010;69(3):571-574.

13. Gerlag DM, Safy M, Maijer KI, Tas SW, Starmans-kool M, van Tubergen A, et al. A Single Infusion of Rituximab Delays the Onset of Arthritis in Subjects at High Risk of Developing RA. Arthritis Rheumatol. 2016;68.

14. Al-Laith M, Jasenecova M, Abraham S, Bosworth A, Bruce IN, Buckley $C D$, et al. Arthritis prevention in the pre-clinical phase of RA with abatacept (the APIPPRA study): a multi-centre, randomised, double-blind, parallel-group, placebo-controlled clinical trial protocol. Trials. 2019;20(1):429.

15. Niemantsverdriet E, Dakkak YJ, Burgers LE, Bonte-Mineur F, SteupBeekman GM, van der Kooij SM, et al. TREAT Early Arthralgia to Reverse or Limit Impending Exacerbation to Rheumatoid arthritis (TREAT EARLIER): a randomized, double-blind, placebo-controlled clinical trial protocol. Trials. 2020;21 (1):862.

16. van Boheemen L, Turk S, Beers-Tas MV, Bos W, Marsman D, Griep EN, et al. Atorvastatin is unlikely to prevent rheumatoid arthritis in high risk individuals: results from the prematurely stopped STAtins to Prevent Rheumatoid Arthritis (STAPRA) trial. RMD Open. 2021;7(1).

17. an Dongen H, van Aken J, Lard LR, Visser K, Ronday HK, Hulsmans $\mathrm{HM}$, et al. Efficacy of methotrexate treatment in patients with probable rheumatoid arthritis: a double-blind, randomized, placebo-controlled trial. Arthritis Rheum. 2007;56(5):14241432.

18. Burgers LE, Allaart CF, Huizinga TWJ, van der Helm-van Mil AHM. Brief Report: Clinical Trials Aiming to Prevent Rheumatoid Arthritis Cannot Detect Prevention Without Adequate Risk Stratification: A Trial of Methotrexate Versus Placebo in Undifferentiated Arthritis as an Example. Arthritis Rheumatol. 2017;69 (5):926-931. 